\title{
Observation of the limit cycle in asymmetric plasma divided by a magnetic filter
}

\author{
Kazuo Ohi, Hiroshi Naitou, ${ }^{\text {a) }}$ Yasushi Tauchi, and Osamu Fukumasa \\ Department of Electrical and Electronic Engineering, Yamaguchi University, Tokiwadai 2-16-1, \\ Ube 755-8611, Japan
}

(Received 18 July 2000; accepted 4 October 2000)

\begin{abstract}
An asymmetric plasma divided by a magnetic filter is numerically simulated by the one-dimensional particle-in-cell code VSIM1D [Koga et al., J. Phys. Soc. Jpn. 68, 1578 (1999)]. Depending on the asymmetry, the system behavior is static or dynamic. In the static state, the potentials of the main plasma and the subplasma are given by the sheath potentials, $\phi_{M} \sim 3 T_{M e} / e$ and $\phi_{S} \sim 3 T_{S e} / e$, respectively, with $e$ being an electron charge and $T_{M e}$ and $T_{S e}$ being electron temperatures $\left(T_{M e}\right.$ $>T_{S e}$ ). In the dynamic state, while $\phi_{M} \sim 3 T_{M e} / e, \phi_{S}$ oscillates periodically between $\phi_{S \text {, } m i n}$ $\sim 3 T_{S e} / e$ and $\phi_{S, \max } \sim 3 T_{M e} / e$. The ions accelerated by the time varying potential gap get into the subplasma and excite the laminar shock waves. The period of the limit cycle is determined by the transit time of the shock wave structure. (c) 2001 American Institute of Physics.
\end{abstract}

[DOI: $10.1063 / 1.1329653]$

\section{INTRODUCTION}

The magnetic filter (MF) is a localized magnetic field, which can divide a plasma into two regions of different parameters. In the volume negative hydrogen/deuterium ion source, for example, the MF is the critical component to isolate a diffused plasma from a source plasma. The major mechanism to create negative hydrogen/deuterium ions is the two-step process of vibrational excitation and dissociative attachment. ${ }^{1,2}$ The source plasma involves fast electrons with energies in excess of $20-30 \mathrm{eV}$ to produce molecules of highly vibrationally excited states. These excited molecules can travel to the diffused plasma across the MF, while high energy electrons are reflected by the MF. The diffused plasma, hence, includes only low energy electrons with a temperature of about $1 \mathrm{eV}$, which are necessary for the dissociative attachment to the vibrationally excited molecules. This sort of "double plasma" configuration might be useful for processing plasma; the plasma with energetic electrons and the low temperature plasma without energetic electrons are needed for the production of radical species and for the reduction of the harmful energetic ion bombardment (due to the sheath potential) to the substrate surface, respectively. ${ }^{3}$

There are three effects of the MF. The first and primary effect is the Lorentz force on electrons and ions. Depending on the mass and the energy of charged particles, the action of the MF is different for individual particles. The MF, hence, works as the filter to select particles which can go through the MF. The second is the diffusion process of the charged particles. Collisional and/or the turbulent transports across the MF can explain the character of the MF in which energetic electrons diffuse much more slowly than the low energy electrons. The turbulent diffusion is due to $\mathbf{E} \times \mathbf{B}$ drifts with $\mathbf{E}$ being the fluctuating electrostatic electric field and $\mathbf{B}$

\footnotetext{
${ }^{a}$ Guest staff of National Institute for Fusion Science, Toki, Gifu 509-5292, Japan (1 April 1999-31 March 2001).
}

being the static magnetic field of the MF; the finite gyroradius effects of electrons elucidate the slow random walks of energetic electrons because high energy electrons average out the fine scale fluctuations of the electric field. ${ }^{4-6}$ The third effect is the formation of the potential gap between two regions separated by the MF. This potential gap made by the first and second effects acts as an electrostatic filter on electrons and ions.

In order to understand the complex behavior of the plasma with the MF, particle-in-cell (PIC) simulations have been executed. The transport process and the potential formation of the plasma divided by the MF have been investigated by two-dimensional particle simulation. ${ }^{5-8}$ We have believed in the existence of the static state with the equilibrium density, temperature, and space potential profiles. So our concern was limited to the understanding of the transport process due to the fluctuating turbulent electrostatic field around the MF. Recently, we unexpectedly found the nonstatic dynamic state in the plasma with the $\mathrm{MF}^{9}$ by executing the one-dimensional PIC simulation with a newly developed VSIM1D code ${ }^{10}$ (visualized particle simulation code in one dimension) which runs on a PC-UNIX operating system and displays the real time portrayal of the phase space plots of charged particles and the potential profile, etc., on the monitor. The limit cycle in the terminology of nonlinear physics is observed. The periodic variation of the potential gap and the periodic excitation of the laminar shock waves ${ }^{11}$ caused by ions accelerated by the potential gap constitute the physics underlying the limit cycle. The movies of the temporal evolutions of the potential profiles and the phase space plots of ions can be found on the CD-ROM compiled in Ref. 12. This article extends the study of Ref. 9 in which only the discovery of the dynamic state with a brief physical discussion was reported. Simulations using a doubled system size with various parameters are done to verify the generality of the previous results. Detailed measurements are added to clear the 


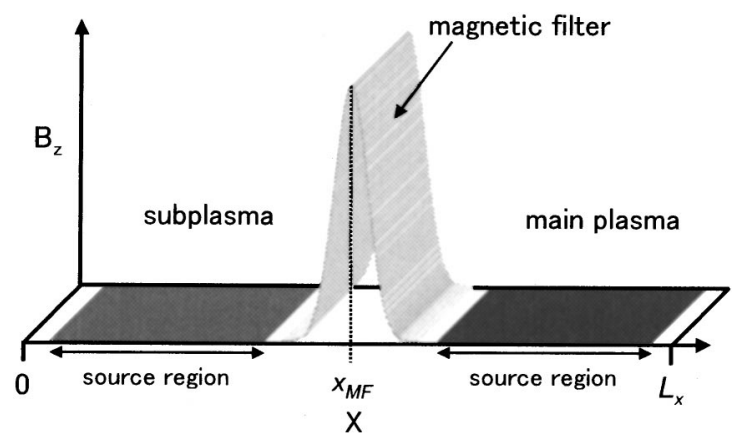

FIG. 1. Schematic diagram of the simulation model with the MF.

physical picture of the phenomena. Also, the parameter range in which the system behaves dynamically is studied carefully.

This paper is organized as follows. The basic simulation model of the asymmetric plasma with the MF is described in Sec. II. Simulation results are presented in Sec. III. Conclusions and a discussion are given in Sec. IV.

\section{SIMULATION MODEL}

The one-dimensional particle-in-cell code $\operatorname{VSIM}_{10}{ }^{10}$ is used for the simulation of the asymmetric plasma divided by the MF. Physical quantities are allowed to change only in the $x$ direction. Hydrogen plasma is assumed. Full dynamics of electrons and ions are followed under the electrostatic approximation. A schematic diagram of the simulation model is depicted in Fig. 1. The left and right ends of the system, $x$ $=0$ and $x=L_{x}$, are grounded walls. Particles hitting the walls are absorbed there. Ion sheaths, hence, will be formed next to the walls. There is a MF at the center of the system $\left(x=x_{\mathrm{MF}}\right)$ and the direction of the magnetic field is in the $z$ direction. The spatial profile of the magnetic field strength is given by

$$
B(x)=B_{0} \exp \left[-0.5\left(x-x_{\mathrm{MF}}\right)^{2} / a_{\mathrm{MF}}^{2}\right],
$$

where $B_{0}$ is the maximum magnetic field strength and $a_{\mathrm{MF}}$ is the characteristic width of the MF. The strength of the MF is chosen to influence only electron dynamics; ions move freely across the MF. To the right of the MF, there is a high temperature and high density main plasma. A low temperature and low density subplasma exists to the left of the MF. The source regions are located in the main plasma and the subplasma, respectively, to produce new electrons and ions, which will equal the particle loss to the walls.

The physical quantities in this paper are expressed in normalized units. The length is normalized by the grid size $\Delta$. The time is normalized by the inverse of the electron plasma angular frequency $\omega_{p e}^{-1}$, where $\omega_{p e}=\left(n_{e 0} e^{2} /\right.$ $\left.\epsilon_{0} m_{e}\right)^{1 / 2}$ is defined by the initial average electron density $\left(n_{e 0}=n_{i 0}=n_{0}\right)$ in the main plasma, $e$ and $m_{e}$ are the charge and the mass of electrons, and $\epsilon_{0}$ is the permittivity in vacuum. Temperatures and potentials are normalized by $m_{e} \Delta^{2} \omega_{p e}^{2}$ and $m_{e} \Delta^{2} \omega_{p e}^{2} / e$, respectively. Note that temperatures in this paper are expressed in the energy unit. The normalized strength of $B_{0}$ equals $\omega_{c e} / \omega_{p e}\left(\omega_{c e}=e B_{0} / m_{e}\right.$ is the electron cyclotron angular frequency at $\left.x=x_{\mathrm{MF}}\right)$. Simu- lation parameters are as follows: system size: $L_{x}=400, x_{\mathrm{MF}}$ $=200, a_{\mathrm{MF}}=12, B_{0}=0.2-1.0$, electron Debye length in the main plasma: $\lambda_{\mathrm{D} e}=2$, mass ratio: $m_{i} / m_{e}=1836$, time step size: $\Delta t=0.2$, total number of time steps: $N_{t}=400000$. Hereafter, we will add subscripts $M$ and $S$ for the quantities related to the main plasma and the subplasma, respectively. The initial electron temperatures of the respective plasmas are $T_{M e}=4$ and $T_{S e}=1$. The initial ion temperatures are set to be $T_{M i}=T_{S i}=0.4$. The initial electron density of the subplasma is one fourth of the density of the main plasma. The initial ion density profile is the same as that of electrons. Note that initial conditions are not so important in this simulation because the system includes particle source and loss and our concern is the long time behavior.

In order to make up for the particle loss from the respective regions, electrons and ions are injected constantly into the source regions. In the main plasma, one electron and one ion are injected every one time step with given temperatures of $T_{M e}=4$ and $T_{M i}=0.4$ in the region of $220<x<380$. In the subplasma, one electron and one ion are inserted every $N_{\text {in }}$ time steps with $T_{S e}=1$ and $T_{S i}=0.4$ in the region of $20<x<180 . N_{\text {in }}$ is varied between 8 and 256. In the source regions, the artifical "thermalization" is done; velocity distributions of electrons in the respective source regions are reset to form new Maxwellian distributions every 150 time steps. Without this process, the electron velocity distributions would be cooled eventually because only low energy electrons are confined in the system by the sheath potential near the walls. The total number of particles used in this simulation for each species is about 30000 . The total running time is about $12 \mathrm{~h}$ on the PC/AT compatible personal computer using the $\mathrm{CPU}$ of Celeron $450 \mathrm{MHz}$.

\section{SIMULATION RESULTS}

Before presenting the simulation results for the dynamic state, let us consider the static state. It is easy to predict that there are two types of static equilibrium potential profile for the asymmetric plasma divided by the MF. The first corresponds to the case in which the effects of ions coming from the main plasma to the subplasma are negligible. Each plasma has the space potential of the order of the electron temperature $\left(\phi \sim 3 T_{e}\right)$, which is equal to the sheath potential adjacent to the respective grounded wall. The space potential of the main plasma is higher than that of the subplasma, since $T_{M e}>T_{S e}$. The example of this case is shown in Figs. 2 and 3. The parameters chosen for this case are $B_{0}=0.7$ and $N_{i n}=64$. It is found that the system approaches the static state after $t \sim 20000$. Figure 2 represents the time evolutions of the average electron densities in the main plasma and the subplasma. The average electron densities are measured in the source regions of the respective zones. In the stationary state, the average electron density in the main plasma is $\left\langle n_{M e}\right\rangle \simeq 1.66 n_{0}$, while that in the subplasma is $\left\langle n_{S e}\right\rangle$ $\simeq 0.12 n_{0}$. The density ratio $\left\langle n_{S e}\right\rangle /\left\langle n_{M e}\right\rangle \simeq 0.072$ is much larger than the ratio of the particle supplement, $1 / N_{\text {in }}$ $\simeq 0.016$. The improved electron confinement in the subplasma is explained by the ions coming from the main plasma. Figure 3 depicts the time evolutions of the potentials 


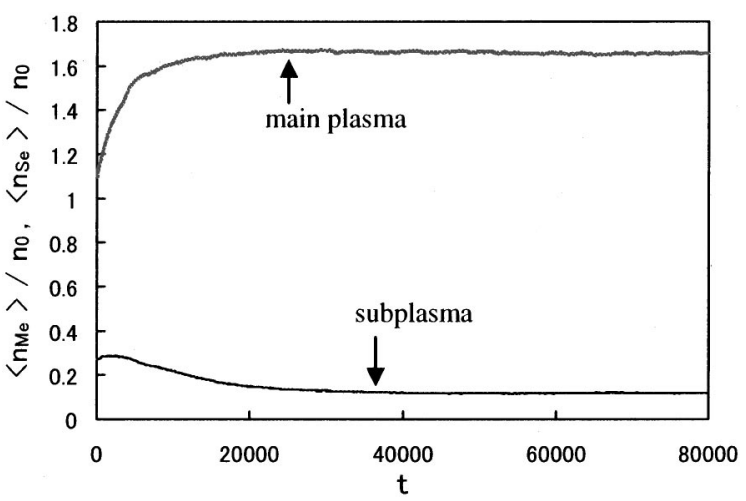

FIG. 2. Time evolutions of average electron densities in the main plasma and the subplasma, $\left\langle n_{M e}\right\rangle$ and $\left\langle n_{S e}\right\rangle$, for $B_{0}=0.7$ and $N_{i n}=64$.

of the main plasma and the subplasma, $\phi_{M}$ and $\phi_{S}$, measured at the centers of the respective regions. These potentials are time averaged over $\tau=20$ to eliminate the fluctuating noise concerning electron plasma oscillations. The potential gap between $\phi_{M}$ and $\phi_{S}$ is observed. The measured potentials, $\phi_{M}$ and $\phi_{S}$, are equal to the sheath potential next to the grounded walls, $\sim 3 T_{M e}$ and $\sim 3 T_{S e}$, respectively. The deviation of $\phi$ from the average value is determined by the thermal fluctuations in the respective areas. (We can reduce these fluctuations by drastically increasing the number of particles in the simulation with the expense of computer resources.) It is to be noticed that the potential gap at the MF accelerates ions in the main plasma into the subplasma; there is an ion beam component in the subplasma. No instability is observed due to the ion beam component in this static case.

The second static case will be found when the effects of ions intruding into the subplasma across the MF are dominant. Electrons in the subplasma cannot keep the space potential from going up by reducing the electron loss to the wall. Therefore, the potential of the subplasma is a little bit higher than that of the main plasma, while the potential of the main plasma is determined by the electron temperature; $\phi_{M} \sim 3 T_{M e}$ and $\phi_{S} \sim 3 T_{M e}+3 T_{M i} \sim 3 T_{M e}$ since $T_{M i} \ll T_{M e}$. (In this case, electrons in the subplasma are confined very well by the sheath potential. Hence, in the strict sense, to maintain the stationary state, the loss mechanism of electrons in the subplasma must be taken into account.)

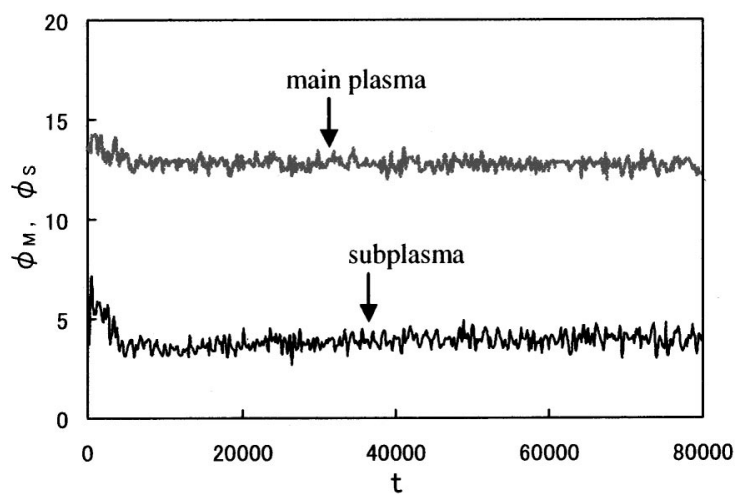

FIG. 3. Time evolutions of potentials at the centers of the main plasma and the subplasma, $\phi_{M}$ and $\phi_{S}$, for $B_{0}=0.7$ and $N_{i n}=64$.

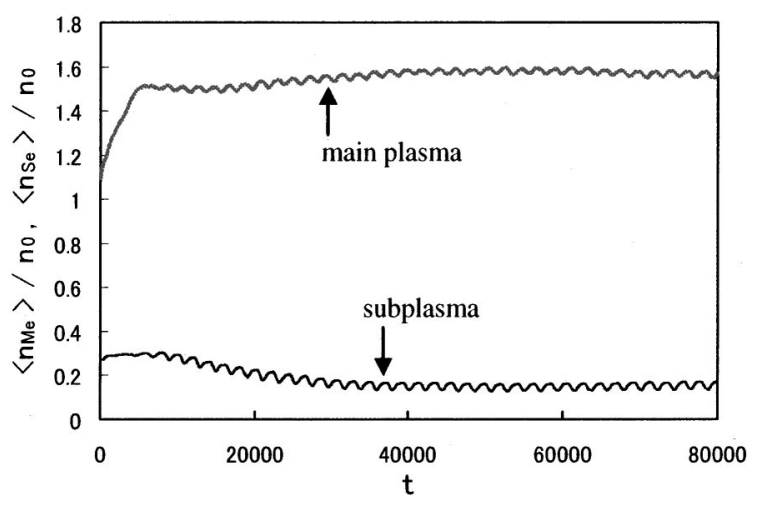

FIG. 4. Time evolutions of average electron densities in the main plasma and the subplasma, $\left\langle n_{M e}\right\rangle$ and $\left\langle n_{S e}\right\rangle$, for $B_{0}=0.5$ and $N_{i n}=64$.

We have found that there is a dynamic state in which the potential in the subplasma oscillates periodically between values of $\sim 3 T_{M e}$ and $\sim 3 T_{S e}$. The example of this dynamic state is shown in Figs. 4 and 5. The parameters chosen for this case are $B_{0}=0.5$ and $N_{i n}=64$. Figure 4 expresses the time evolutions of the average electron densities in the main plasma and the subplasma. We can see the periodic oscillations in the ripples of the average densities in the respective regions after $t \sim 8000$. The system approaches the stationary state after $t \sim 30000$. The period of the oscillations of the ripples is about 2000. In the stationary state, $\left\langle n_{M e}\right\rangle \simeq 1.56 n_{0}$ and $\left\langle n_{S e}\right\rangle \simeq 0.15 n_{0}$ with $\Delta\left\langle n_{M e}\right\rangle \simeq \Delta\left\langle n_{S e}\right\rangle$ $\simeq 0.032 n_{0}$, where $\Delta\left\langle n_{M e}\right\rangle$ and $\Delta\left\langle n_{S e}\right\rangle$ indicate the heights of the density ripples. The density ratio is $\left\langle n_{S e}\right\rangle /\left\langle n_{M e}\right\rangle$ $\simeq 0.096$. Figure 5 depicts the time evolutions of the potentials at the centers of the main plasma and the subplasma. The potential of the main plasma is almost constant $\left(\phi_{M}\right.$ $\sim 12$ ) with small ripples, while that of the subplasma oscillates between $\phi_{S} \sim 3$ and $\phi_{S} \sim 12$. The period of the potential oscillation in the subplasma equals that of the density ripples.

To understand the physical mechanism of the dynamic state, detailed measurements were done concentrating on the case of $B_{0}=0.5$ and $N_{i n}=64$. The typical four periods of the time variations of the average electron densities are extracted from Fig. 4 and shown in Fig. 6. Note that the axes in Fig. 6 are different for $\left\langle n_{M e}\right\rangle$ and $\left\langle n_{S e}\right\rangle$ in the offsets of the scales.

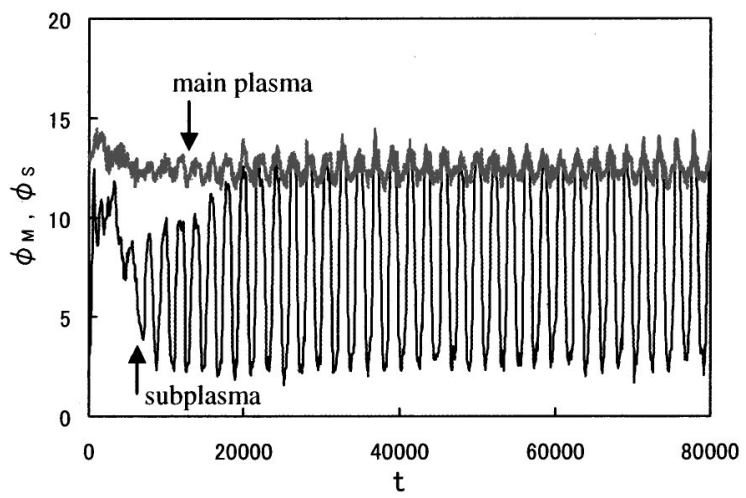

FIG. 5. Time evolutions of potentials at the centers of the main plasma and the subplasma, $\phi_{M}$ and $\phi_{S}$, for $B_{0}=0.5$ and $N_{i n}=64$. 


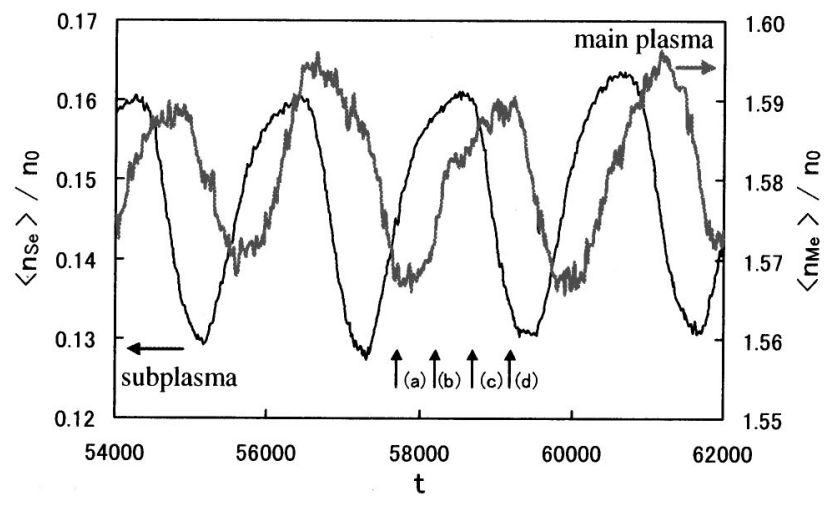

FIG. 6. Typical four periods of average electron density oscillations of the main plasma and the subplasma, $\left\langle n_{M e}\right\rangle$ and $\left\langle n_{S e}\right\rangle$, for $B_{0}=0.5$ and $N_{i n}$ $=64$.

It is clear that $\left\langle n_{S e}\right\rangle$ increases (decreases) when $\left\langle n_{M e}\right\rangle$ decreases (increases) with the delay time of $\tau_{D} \sim 500$. The delay time is caused by the space between source regions, since $\left\langle n_{M e}\right\rangle$ and $\left\langle n_{S e}\right\rangle$ are the average densities of the respective source regions. The ions going out of the source region in the main plasma cross the domain of the MF and come to the source region of the subplasma after the time of $l / v_{D i}$, where $l=40$ is the space between the source regions and $v_{D i}$ is the average drift speed of ions crossing the MF. We can estimate $v_{D i} \sim l / \tau_{D} \sim 0.08$, which is almost the same as the ion drift speed of $v_{D i} \sim 0.1$ given by the normalized equation for the energy conservation,

$$
\frac{1}{2} m_{i} v_{D i}^{2} \sim \phi_{M}-\phi_{S},
$$

where we used $\phi_{M} \sim 12, \phi_{S} \sim 3$, and $m_{i}=1836$.

Typical four periods of the time evolutions of $\phi_{M}$ and $\phi_{S}$ are extracted from Fig. 5 and depicted in Fig. 7. The time scales for Figs. 6 and 7 are the same. We can see that the phase of $\phi_{S}$ is synchronized with that of the density ripple of $\left\langle n_{S e}\right\rangle$, although there is a slight difference in the wave forms of $\phi_{S}$ and $\left\langle n_{S e}\right\rangle$. When $\phi_{S}$ is at the minimum, the ion flux from the main plasma increases due to the potential gap between the main plasma and the subplasma. Then the ion density in the subplasma increases; $\left\langle n_{S e}\right\rangle$ also enhances due to quasineutrality. When $\phi_{S}$ is at the maximum, the ion flux from the main plasma is small since there is no potential gap.

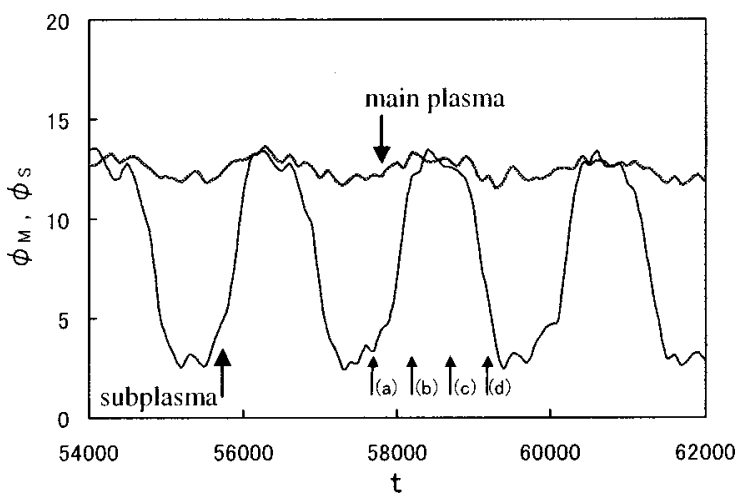

FIG. 7. Typical four periods of potential oscillations at the centers of the main plasma and the subplasma, $\phi_{M}$ and $\phi_{S}$, for $B_{0}=0.5$ and $N_{i n}=64$.
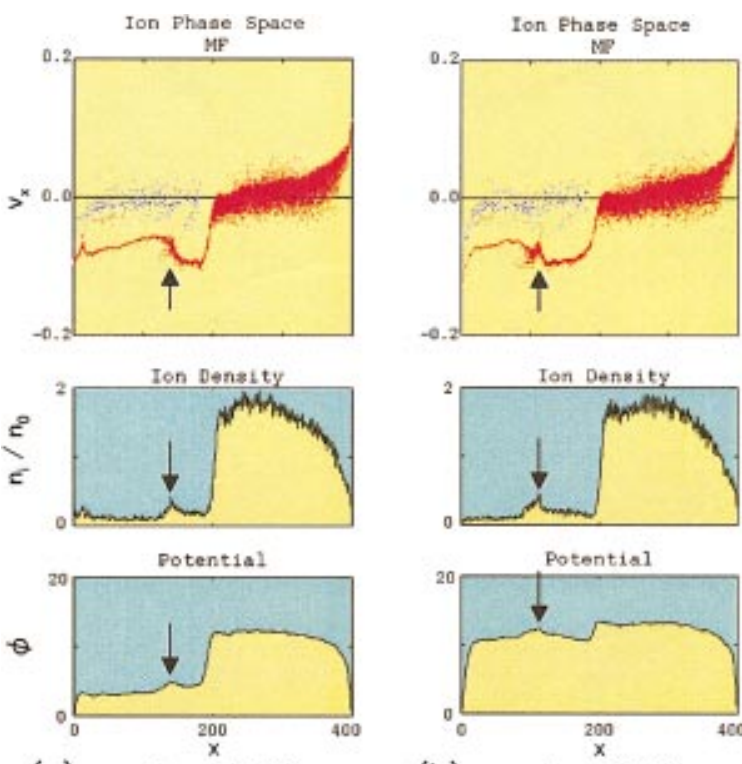

(a) $\quad t=57700$

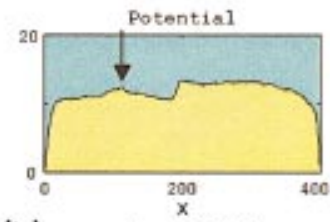

(b)
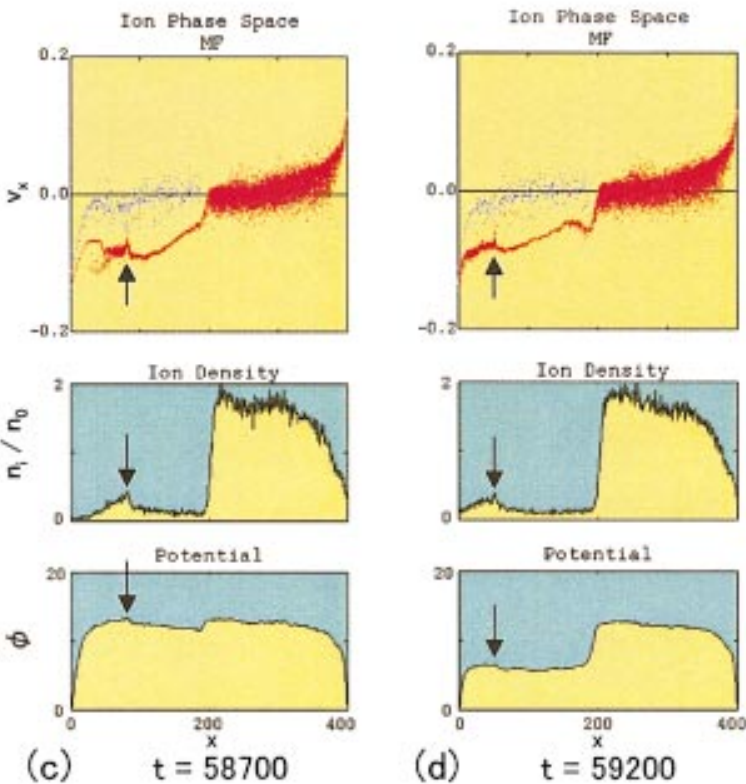

FIG. 8. (Color) Snapshots of phase space plots of ions, ion density profiles, and potential profiles for $B_{0}=0.5$ and $N_{i n}=64$. Time variation for a period is shown.

The part of the slow increase of $\left\langle n_{S e}\right\rangle$ after the fast increase may be explained by the particle supplement in the source region and the good electron confinement due to the large sheath potential at the left wall. As will be shown later, when the potential gap is maximum, the higher energy ion beam with the higher density gets into the subplasma. $\phi_{S}$ and $\left\langle n_{S e}\right\rangle$ reduce when these energetic ions get out of the system. The same process continues periodically.

Snapshots of phase space plots of ions (top), ion density profiles (middle), and potential profiles (bottom) are shown in Fig. 8 for different time steps in one period. Four different times in Fig. 8 are depicted in Figs. 6, 7, 9, and 10 by the arrows with the letters (a), (b), (c), and (d). In the phase space plots, red points indicate ions generated in the main plasma, whereas blue points denote ions introduced in the 


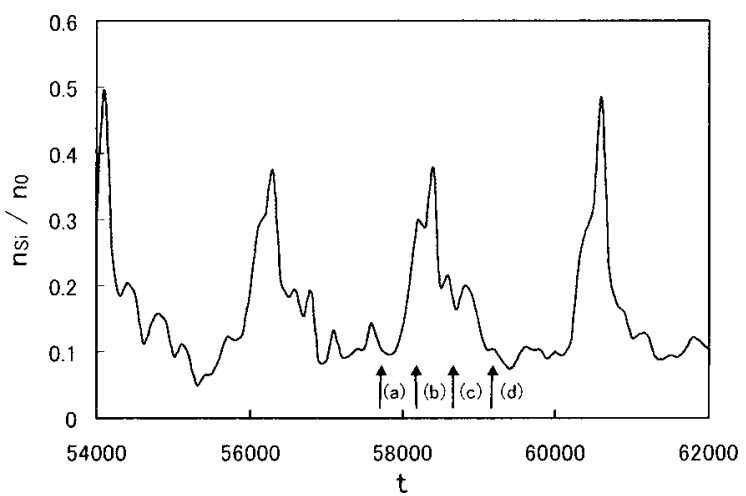

FIG. 9. Typical four periods of the time evolution of the ion density at the center of the subplasma for $B_{0}=0.5$ and $N_{i n}=64$.

subplasma. Hence the red points in the subplasma show ions coming from the main plasma. We can see the structure in the ion density profile in the subplasma. In the density profile, there is a peak moving in the negative $x$ direction. Arrows in Fig. 8 show the positions of the density peaks. The structure of the ion density reflects the phase space dynamics of the ion beam accelerated by the potential gap and traveling in the subplasma. We can also see slight peaks of the electrostatic potential profile at the same positions of the density peaks in the subplasma. The period of the limit cycle is determined by the transit time of the faster ions accelerated when the potential gap is maximum.

In Ref. 9, we called the collective phenomena induced by the ion beam the beam instability. Lately we have recognized that we are observing the laminar shock waves generated by the modulated ion beam. The linear two stream instability is stable for our case. The phenomenon is very close to the one experimentally observed by Ikezi et al. ${ }^{11}$ They used a double plasma device in which the energy of the ion beam coming into the low density target plasma is suddenly increased at some time. The faster ions overtake the slower ions and the shock wave structure is formed. The change of energy in the experiment of Ikezi et al. corresponds to the variation of the potential gap in our case. Both cases are similar in two respects: (1) a significant amount of ions is reflected from and transmitted through the shock front, (2)

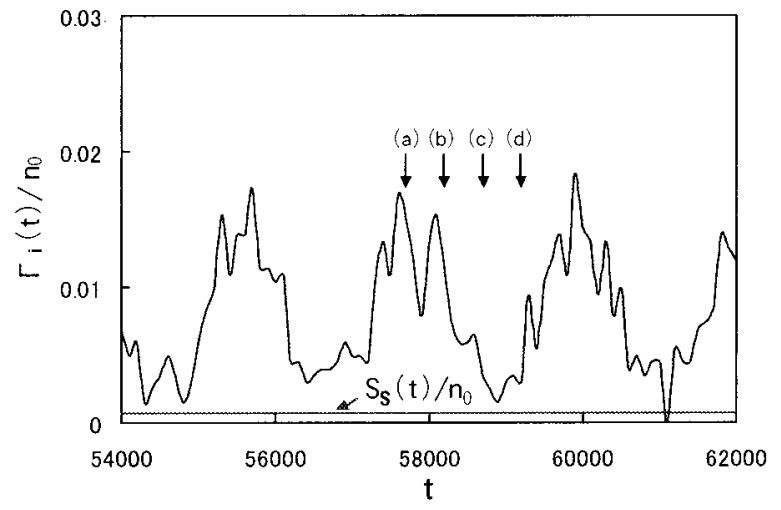

FIG. 10. Typical four periods of the ion flux $\Gamma_{i}$ at the MF penetrating from the main plasma and the subplasma for $B_{0}=0.5$ and $N_{i n}=64$. the width of the shock increases in time. These facts are clearly observed in the phase space plots in Fig. 8. The behavior of the system in one period of the oscillation is summarized in the following. When the potential gap is large, the ion beam flux from the main plasma is large and forms the shock wave structure due to the overtaking of the slower ions by the faster ions [see Fig. 8(a)]. As a result of the higher ion beam flux the potential of the subplasma increases to the level of the potential of the main plasma [Figs. 8(b) and 8(c)]. In this time scale, the ion beam flux from the main plasma reduces as the potential gap decreases. At the time when the head of the shock wave structure approaches the left wall, the potential in the subplasma starts to decrease [Fig. 8(d)]. When the old shock wave goes out of the system, the ions with the higher speed and the higher density come into the subplasma and excite the new shock wave; this process continues periodically.

The speed of the ion sound wave in our simulation is $c_{s}=\sqrt{\left(T_{e}+3 T_{i}\right) / M_{i}} \simeq 0.035$. From the phase space plots of Fig. 8(a) we see that the velocities before and behind the shock wave structure are $v_{\min } \simeq 0.060$ and $v_{\max } \simeq 0.094$, respectively. The velocities of the head and the tail of the shock wave structure are calculated from the 16 snapshots of the ion density profile in one period. The velocity of the head of the shock wave structure is $v_{H} \simeq 0.096$, while the velocity of the tail of the shock wave is $v_{T} \simeq 0.053$. Hence, the width of the shock wave structure increases in time. Note that the arrows in Fig. 8 designate the positions of the tails of the shock wave structures. The ion density between the head and the tail of the shock wave structure is higher than that of the ambient plasma. We can think of the head and tail of the shock wave structure as two shock fronts; in the frame moving with the average ion velocity, we have two shock waves propagating in forward and backward directions. The mach number of the faster shock (head) is $\left(v_{H}-v_{\min }\right) / c_{s} \simeq 1.0$, while that of the slower shock (tail) is $\left(v_{\max }-v_{T}\right) / c_{s} \simeq 1.2$. The difference in mach number may explain why the tail of the shock wave structure is more prominent than the head. The mach number of the head becomes subsonic in time; then the shock wave structure around the head disappears [see Figs. 8(c) and 8(d)].

The time evolution of the ion density at the center of the subplasma is shown in Fig. 9 for four periods of the limit cycle. The time scale of Fig. 9 is the same as those of Figs. 6 and 7. The sharp peaks in Fig. 9 correspond to the passage of the tail of the shock wave structure. The sudden reduction of the ion density just after the maximum is followed by the slow decrease. Considering that the ion density displayed in Fig. 9 is the sum of the background ions born in the subplasma and the ions coming from the main plasma, the amount of the ions coming from the main plasma is an order of magnitude larger than that of the background ions in the subplasma.

The time evolution of the ion flux $\Gamma_{i}$ at the MF for the typical four periods is shown in Fig. 10. The ions crossing the MF from the main plasma to the subplasma are counted and time averaged over $\tau=20$. The quantity depicted in the vertical axis, $\Gamma_{i} / n_{0}$, is the number of counts per one normalized time divided by the initial number of ions per one grid 


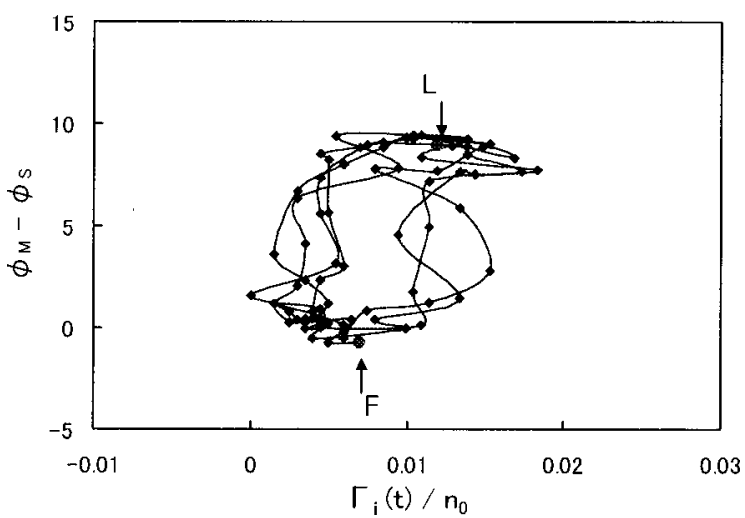

FIG. 11. Lissajous figure of the potential gap, $\phi_{M}-\phi_{S}$, vs the ion flux $\Gamma_{i}$ at the MF for $B_{0}=0.5$ and $N_{i n}=64$. Typical four periods are shown. F and $\mathrm{L}$ designate the first and last data, respectively. Data rotate in the clockwise direction.

in the main plasma. The number of ions (electrons) supplied in the source region in the subplasma per one normalized time, $S_{S}$, is also shown in Fig. 10. The effect of supplied ions is not critical (except for neutralizing the supplied electron charges), although supplied electrons have a crucial role to keep the plasma in the subplasma. The relation between $\Gamma_{i}$ and the potential gap, $\phi_{M}-\phi_{S}$, is summarized in the Lissajous figure in Fig. 11. Only four periods corresponding to Figs. 7 and 10 are shown in the figure. The data in the Lissajous figure rotate in the clockwise direction. When $\Gamma_{i}$ is maximum (minimum) the potential gap reduces (increases).

It is natural that when the potential gap is maximum, $\Gamma_{i}$ starts to increase. If $\Gamma_{i}$ at the MF is larger than the ion flux to the left wall, the electron flux to the left wall tends to decrease to keep the charge neutrality. Because only high energy electrons can reach the left wall, the number of electrons to prevent the space potential from going up is limited. So, if the imbalance of $\Gamma_{i}$ at the MF and the left wall (which might be proportional to the $\Gamma_{i}$ at the MF) increases beyond some value, electrons cannot contribute to keep the charge neutralization. Once the sheath potential adjacent to the left wall becomes high enough, no electrons can reach the left wall and the space potential in the subplasma increases up to the space potential of the main plasma. The maximum of $\phi_{S}$ is limited by $\phi_{M}$, because $\Gamma_{i}$ changes sign if $\phi_{S}$ is greater than $\phi_{M}$.

On the other hand, it is difficult to explain what triggers the buildup of the potential gap. This is related to the problem of why the successive pulse does not appear before the former pulse reaches very close to the wall. We only see that $\Gamma_{i}$ at the MF is minimum in the increasing phase of the potential gap. We have verified that the period of the limit cycle is determined by the transit time of the shock wave structure by doing runs with $L_{x}=200,400,800,1600$. Hence we can conclude that the shock wave structure approaching the wall triggers the increase of the potential gap. Figure 12 shows the Lissajous figure of the potential gap versus the position of the faster shock front (head) for the typical four periods. We cannot measure the position of the shock fronts (head and tail) when the high energy ions are close to the MF. If the position of the shock front is extrapolated to this

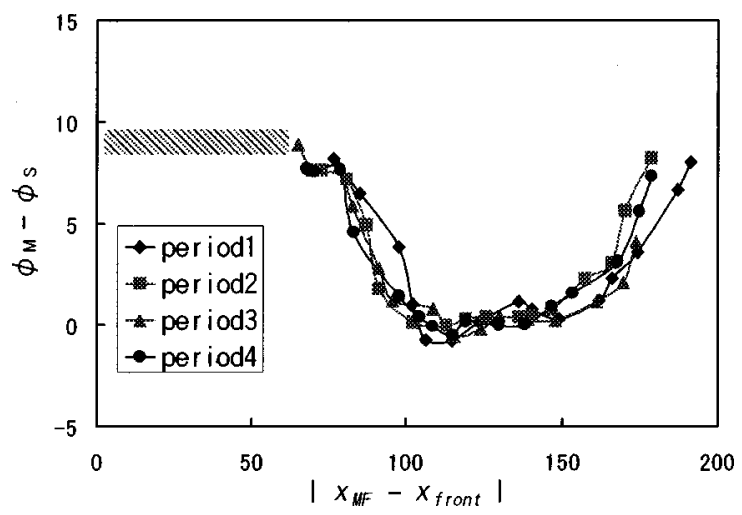

FIG. 12. Lissajous figure of the potential gap, $\phi_{M}-\phi_{S}$, vs distance of the faster shock front (head) from the MF for $B_{0}=0.5$ and $N_{i n}=64$. Typical four periods are shown.

region, the data are expected to be in the hatched region in Fig. 12. The potential gap is maximum before the shock is formed. As the shock front propagates in the subplasma, the potential gap disappears once and builds up later again. It is interesting that the potential gap increases before the head reaches the wall when the shock wave structure is very close to the wall. This may be explained in the following. There is an ion sheath without electrons adjacent to the left wall when $\phi_{S}$ is maximum. In front of the shock wave structure, there is an electron sheath (the electron density is higher than the ion density). The shock wave has the effect of pushing the electrons in front of the shock wave structure to the ion sheath at the wall. So the sheath structure near the wall changes drastically; the space potential of the subplasma decreases to the minimum value. The minimum of $\phi_{S}$ is limited by the sheath potential determined by the electron temperature because the bulk electrons prevent the potential from going down further by enhancing the electron flux to the wall.

To identify the parameter range in which the asymmetric plasma with the MF behaves dynamically, simulations with different values of $N_{i n}$ and $B_{0}$ are done. Figure 13 depicts the average electron density in the subplasma $\left\langle n_{S e}\right\rangle$ depending on the rate of the particle supplement in the subplasma, $1 / N_{i n}$. The strength of the MF is fixed at $B_{0}=0.5$. For $B_{0}$ $=0.5$, the electrons in the main plasma and the subplasma

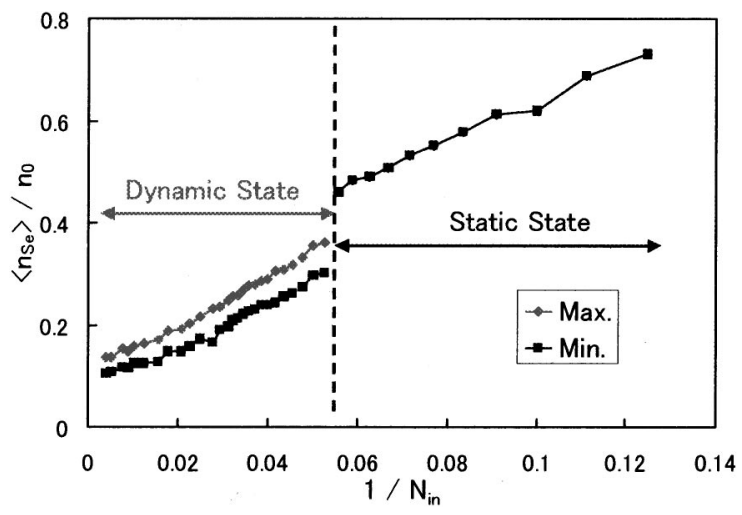

FIG. 13. Average electron density in the subplasma $\left\langle n_{S e}\right\rangle$ depending on the rate of particle supplement in the subplasma, $1 / N_{i n}$. The maximum and minimum values are shown for the dynamic case. 


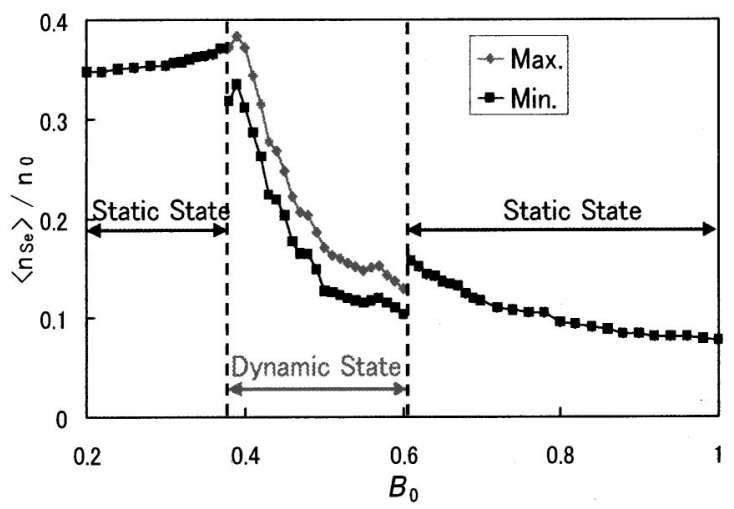

FIG. 14. Average electron density in the subplasma $\left\langle n_{S e}\right\rangle$ depending on $B_{0}$. The maximum and minimum values are shown for the dynamic case.

cannot commute each other. The maximum and minimum values are shown in Fig. 13. The cases with the same values of maximums and minimums correspond to the static states. The dynamic state of the limit cycle is observed for $1 / N_{\text {in }}$ $\leqslant 1 / 19$, while the static state is seen for $1 / N_{i n} \geqslant 1 / 18$. It is to be noted that there is a discontinuity between $1 / N_{i n}=1 / 19$ and $1 / 18$. As $1 / N_{\text {in }}$ reduces, the solution of this nonlinear system changes from the static state to the dynamic state with finite radius of the limit cycle. In the dynamic state, we believe that the positive feedback has the critical effect before the stationary dynamic state is formed. Initially, we assume a static state. Due to the thermal noise, the potential gap is modulated slightly. The weak shock wave produced by the velocity modulated ion beam gets into the left wall. If the reduction of the space potential caused by the approaching shock wave is bigger than that of the former potential modulation, the stronger shock wave is formed. This process continues until the maximum potential of the subplasma goes up to $\phi_{M}$. We can see this positive feedback phase in Fig. 5 for $500 \leqslant t \leqslant 2000$. If the effect of the approaching shock wave is mitigated by bulk electrons, there is no positive feedback and the static state remains. So the threshold exists for the bifurcation of states, which may be the ratio of the ion beam flux from the main plasma to the plasma density in the subplasma. Hence the dynamic state manifests for the low value of $1 / N_{\text {in }}$, which is equivalent to the low plasma density in the subplasma. The periods of the limit cycles are the same for all cases with $1 / N_{i n} \leqslant 1 / 19$.

Figure 14 shows the average electron density in the subplasma, $\left\langle n_{S e}\right\rangle$, depending on $B_{0}$. The parameter of $N_{i n}$ $=64$ is fixed. The discontinuity in the data is also observed in this case. For the stronger magnetic field of $B_{0} \geqslant 0.61$, the static state with the potential gap is observed. Although there is no communication of electrons between the main plasma and the subplasma for $B_{0} \gtrsim 0.5$, the electron density at the $\mathrm{MF}$ is determined by the electrons coming from the main plasma or the subplasma. The penetration length of electrons into the region of the MF is roughly determined by the Larmor radius, hence the electron density at the MF is a decreasing function of $B_{0}$. Due to the quasineutrality, the ion density at the MF is also a decreasing function of $B_{0}$; the ion flux from the main plasma to the subplasma reduces as $B_{0}$ increases. So, for the larger value of $B_{0}$, the ion beam from the main plasma does not have enough power to cause the potential increase in the subplasma. For the weaker magnetic field of $B_{0} \leqslant 0.37$, the static states with $\phi_{S} \sim 3$ and $\phi_{M} \sim 12$ are observed again. For these lower values of $B_{0}$, energetic electrons in the main plasma pass the MF, lose energy due to the potential gap, and enter into the subplasma. So the electron density in the subplasma increases. The increased number of electrons in the subplasma has the function to keep the sheath potential at the level of $\phi_{S} \sim 3$.

We observe the dynamic state for $0.38 \leqslant B_{0} \leqslant 0.6$. For $0.46 \leqslant B_{0} \leqslant 0.6$, the system shows the limit cycle. For the relatively weak values of $0.38 \leqslant B_{0} \leqslant 0.45$, the intermittent states manifest. In the intermittent cases, some of electrons from the main plasma can go to the subplasma and affects the potential change in the subplasma. The study of these intermittent states is outside the scope of this paper and will be the subject of a future paper.

\section{CONCLUSIONS AND DISCUSSION}

The asymmetric plasma divided by the magnetic filter (MF) is numerically simulated by the one-dimensional particle-in-cell code VSIM1D. The strength of the MF is chosen to influence only electron dynamics; ions move freely across the MF. The main plasma with the high temperature $\left(T_{M e}\right)$ and the high density faces the subplasma with the low temperature $\left(T_{S e}\right)$ and the low density across the MF located at the center of the system. The particles hitting the grounded walls at the left and right ends of the system are absorbed there; there are ion sheaths adjacent to the walls. The fact that we are treating the bounded system is important because the effect of the sheath potential is crucial to the observed phenomena. Depending on the asymmetry, the system's behavior is static or dynamic. In the static state, the potentials of the main plasma and the subplasma are given by the sheath potentials, $\phi_{M} \sim 3 T_{M e}$ and $\phi_{S} \sim 3 T_{S e}$, respectively. In the dynamic state, while the potential in the main plasma is almost constant with $\phi_{M} \sim 3 T_{M e}$, the potential in the subplasma oscillates periodically between $\phi_{S, \min } \sim 3 T_{S e}$ and $\phi_{S, \max } \sim 3 T_{M e}$. When the potential gap between the main plasma and the subplasma is maximum, the ion beam with the higher speed and the higher density gets into the subplasma from the main plasma. Then the potential in the subplasma gradually increases because of the increased ion beam flux from the main plasma. The ion beam with the higher speed excites the laminar shock wave when it travels in the subplasma. When the shock wave structure is very close to the left wall, the space potential of the subplasma becomes small. This may be explained in the following. When the shock wave structure is approaching the wall, there is an ion sheath without electrons adjacent to the wall. The electrons in front of the shock being pushed into the ion sheath change the sheath structure and reduce the space potential in the subplasma. Due to the rebuilt large potential gap, the new ion beam with the higher speed and the higher density gets into the subplasma across the MF. This process continues periodically. The period of the limit cycle is deter- 
mined by the transit time of the shock wave structure. The radius of the limit cycle for the potential gap is equal to $\sim 0.5\left(3 T_{M e}-3 T_{S e}\right)$.

The phenomena presented in this paper can be understood as the self-sustained oscillation related to the bifurcation of the potential in the subplasma (or the electric field at the MF). The phenomena caused by the transitions between two bifurcated states have been observed in toroidal magnetic fusion devices. One example is the edge localized modes (ELMs) in tokamaks. ${ }^{13}$ Dithering ELMs are recognized as the transitions between $L$ mode and $H$ mode in which $L$ and $H$ mean low and high confinements, respectively. The experimentally observed dithering ELMs can be elucidated by a limit cycle solution of the Ginzburg-Landautype equation. ${ }^{14}$ The other example is a self-sustained oscillation in the CHS heliotron/torsatron device related to the bifurcation of a radial electric field and the formation of a transport barrier. ${ }^{15}$ Sometimes the bifurcated system behaves chaotically or intermittently as in the case of ELMs. ${ }^{16,17} \mathrm{We}$ have also observed the dynamic state with intermittency, although the presentation of details will be the subject of a forthcoming paper.

\section{ACKNOWLEDGMENTS}

The authors would like to express their thanks to Professor T. Kamimura, Professor K. Itoh, and Professor $\mathrm{H}$. Sanuki, National Institute for Fusion Science; Professor Y. Kawai, Kyushu University; Professor K. Saeki, Shizuoka University; and Professor Y. Ohsawa, Nagoya University for stimulating discussions.
This work was supported in part by a Grant-in-Aid for Scientific Research from the Japanese Ministry of Education, Science, Sports and Culture.

${ }^{1}$ J. R. Hiskes and A. M. Kano, J. Appl. Phys. 56, 1927 (1984).

${ }^{2}$ O. Fukumasa, J. Phys. D 22, 1668 (1989).

${ }^{3}$ O. Fukumasa, Y. Tauchi, and S. Sakiyama, J. Appl. Phys. 36, 4593 (1997).

${ }^{4}$ H. Naitou, T. Kamimura, and J. M. Dawson, J. Phys. Soc. Jpn. 46, 258 (1979).

${ }^{5}$ O. Fukumasa, H. Naitou, and S. Sakiyama, J. Appl. Phys. 74, 848 (1993).

${ }^{6}$ H. Naitou, O. Fukumasa, and K. Sakachou, Rev. Sci. Instrum. 67, 1149 (1996).

${ }^{7}$ H. Naitou, O. Fukumasa, K. Sakachou, and K. Mutou, Rev. Sci. Instrum. 65, 1438 (1994).

${ }^{8}$ H. Naitou, O. Fukumasa, K. Sakachou, and K. Mutou, Fusion Eng. Des. 26, 523 (1995).

${ }^{9}$ H. Naitou, K. Ohi, and O. Fukumasa, Rev. Sci. Instrum. 71, 875 (2000).

${ }^{10}$ K. Koga, H. Naitou, and Y. Kawai, J. Phys. Soc. Jpn. 68, 1578 (1999).

${ }^{11}$ H. Ikezi, T. Kamimura, M. Kako, and K. E. Lonngren, Phys. Fluids 16, 2167 (1973).

${ }^{12}$ H. Naitou, K. Ohi, Y. Tauchi, and O. Fukumasa, J. Plasma Fusion Res. 76, 3 (2000).

${ }^{13}$ H. Zohm, Plasma Phys. Controlled Fusion 38, 105 (1996).

${ }^{14}$ S.-I. Itoh, K. Itoh, A. Fukuyama, Y. Miura, and the JFT-2M Group, Phys. Rev. Lett. 67, 2485 (1991).

${ }^{15}$ A. Fujisawa, H. Iguchi, H. Idei, S. Kubo, K. Matsuoka, S. Okamura, K. Tanaka, T. Minami, S. Ohdachi, S. Morita, H. Zushi, S. Lee, M. Osakabe, R. Akiyama, Y. Yoshimura, K. Toi, H. Sanuki, K. Itoh, A. Shimizu, S. Takagi, A. Ejiri, C. Takahashi, M. Kojima, S. Hidekuma, K. Ida, S. Nishimura, M. Isobe, N. Inoue, R. Sakamoto, S.-I. Itoh, Y. Hamada, and M. Fujiwara, Phys. Rev. Lett. 81, 2256 (1998).

${ }^{16}$ S.-I. Itoh, S. Toda, M. Yagi, K. Itoh, and A. Fukuyama, Plasma Phys. Controlled Fusion 40, 737 (1998)

${ }^{17}$ S. Toda, S.-I. Itoh, M. Yagi, K. Itoh, and A. Fukuyama, J. Phys. Soc. Jpn. 68, 3520 (1999) 